\title{
Presencia de la familia Hymenophyllaceae (Pteridophyta) en el Parque Katalapi, Cordillera de Quillaipe, Provincia de Llanquihue, Chile
}

\section{Presence of Hymenophyllaceae family (Pteridophyte) in the Katalapi Park, Cordillera de Quillaipe, Llanquihue Province, Chile}

\author{
Maria José Parra ${ }^{1 *}$, Karina Acuña ${ }^{2}$, Luis Corcuera² \& Roberto Rodríguez ${ }^{2}$ \\ ${ }^{1}$ Centro de Biotecnología Gran Concepción, Universidad Andrés Bello, Autopista Concepción-Talcahuano s/n. Talcahuano \\ ²Departamento de Botánica, Universidad de Concepción, Casilla 160-C, Concepción, Chile. \\ *mjparramorraga@gmail.com
}

\begin{abstract}
Three Hymenophyllaceae genera were found in Katalapi Park, Region de Los Lagos, Chile: Hymenoglossum, Serpyllopsis, and Hymenophyllum. While the first two genera are monotypic, the third one is represented by 16 species. In this study, we propose that the Hymenophyllum species that were not found in the area are doubtful for Chile due to the inconsistency of their diagnostic characters. From the conservation point of view of this group, six of the species in the park are classified as vulnerable. Finally, and based on vegetative characters, an identification key for Hymenophyllum genera is given.
\end{abstract}

La familia Hymenophyllaceae Link posee una distribución cosmopolita, aunque predomina en los trópicos y en las áreas templadas del Hemisferio Sur. Muestra una marcada diversidad en términos de morfología y requerimiento de hábitat. Entre las características más llamativas se destaca la presencia de frondes uniestratificadas, esporas con clorofila y la ausencia de estomas (Tryon \& Tryon 1982). Esta familia presenta dificultades para una clara y precisa clasificación sistemática, ya que sus caracteres diagnósticos son poco constantes, observándose muchas variedades, formas intermedias y, en ocasiones, poca delimitación entre los grupos. Por esto, existen grandes discrepancias sobre la interpretación de tales caracteres al momento de presentar una clasificación.

En la Flora de Chile, Rodríguez (1995) reconoce 4 géneros presentes en el país: Trichomanes L., Serpyllopsis Bosch, Hymenoglossum C.Presle Hymenophyllum Sm. Trichomanes presenta 3 especies, dos endémicas del Archipiélago de Juan Fernández y una tercera que crece en dicho archipiélago y Chile continental. Serpyllopsis e Hymenoglossum son géneros monoespecíficos y se encuentran en los bosques subantárticos de Chile y Argentina y Juan Fernández. El género Hymenophyllum es el más abundante y diverso en el país con 19 especies, 18 de ellas continentales y 1 endémica del Archipiélago de Juan Fernández.

Esta familia de pteridófitos es una de las más diversas del país (Godoy \& Figueroa 1989, Ponce et al. 2002) y se distribuye desde los $34^{\circ} \mathrm{S}$ de modo continuo a los $55^{\circ} \mathrm{S}$, presentando su máxima riqueza entre los $40^{\circ}$ y $43^{\circ} \mathrm{S}$. Sin embargo, se ha hecho muy difícil hacer una delimitación definitiva de los rangos de sus especies, por la dificultad de su determinación. Debido a que los individuos de esta familia son un elemento higrófilo, su abundancia aumenta a partir de los $37^{\circ} \mathrm{S}$, donde las precipitaciones aumentan con la latitud, generando un exuberante bosque siempreverde (Grau 1995).

El objetivo de nuestro trabajo es evaluar la diversidad de la familia "in situ” en un área potencial de máxima riqueza y generar una clave taxonómica de fácil uso basada sólo en caracteres vegetativos.

El área de estudio fue el parque privado Katalapi emplazado en los faldeos de la Cordillera de Quillaipe (4131'12.0'S - 7245'02.3'O), ubicada dentro de la Ecoregión del Bosque Templado Lluvioso Valdiviano. El clima del parque es templado con precipitaciones 220 días al año aproximadamente, las que alcanzan los $1.900 \mathrm{~mm}$, además, dada la cercanía del mar las temperaturas máximas y mínimas son moderadas (Reyes-Díaz et al. 2005). Esta área es de reconocida importancia a nivel mundial por su alto grado de endemismo. Los ambientes presentes incluyen bosque nativo maduro y renovales, además de un río que atraviesa la parte baja del parque. El bosque está conformado principalmente por: Drimys winteri J.R.Forst. et G.Forst., Nothofagus nitida (Phil.) Krasser, Weinmannia trichosperma Cav., Amomyrtus luma (Molina) D.Legrand et Kausel, Amomyrtus meli D.Legrand et Kausel, Luma apiculata (DC.) Burret, Eucryphia cordifolia Cav. y Laureliopsis philippiana (Looser) Schodde, entre otras.

Para evaluar la diversidad de especies en el área se efectuaron 4 temporadas de colecta: primavera y verano 
de 2007 e invierno y primavera de 2008. Se realizaron exhaustivas recolecciones, y los ejemplares fueron prensados y trasladados al Departamento de Botánica de la Universidad de Concepción, donde se realizó la determinación de las especies utilizando claves taxonómicas, descripciones originales y su comparación con los ejemplares depositados en el herbario CONC.
Las Hymenophyllaceae del Parque Katalapi están representadas por 3 géneros: Hymenoglossum, Serpyllopsis e Hymenophyllum. Los dos primeros monotípicos e Hymenophyllum con 16 especies (Tabla I).

TABLA I. Especies, distribución y Estado de Conservación de las Hymenophyllaceae presentes en el Parque Katalapi (V: Vulnerable; FP: Fuera de Peligro; IC: Insuficientemente conocida; SC: Sin clasificación) (Baeza et al. 1998).

TABLE I. Species, distribution and conservation status of Katalapi Park Hymenophyllaceae (V: Vulnerable; FP: not in danger; IC: Insufficiently known; SC: not classified) (Baeza et al. 1998).

\begin{tabular}{|c|c|c|}
\hline ESPECIE & $\begin{array}{l}\text { DistRIBUCIÓN } \\
\text { EN CHILE }\end{array}$ & $\begin{array}{c}\text { ESTADO DE } \\
\text { CONSERVACIÓN }\end{array}$ \\
\hline Hymenoglossum cruentum (Cav.) C.Presl & VII-XII, JF & $\mathrm{V}$ \\
\hline $\begin{array}{l}\text { Hymenophyllum caudiculatum Mart. var. productum (C.Presl). } \\
\text { C.Chr. }\end{array}$ & VIII-XII, JF & V \\
\hline H. cuneatum Kunze & VIII-XII, JF & $\mathrm{V}$ \\
\hline H. darwinii Hook.f. ex Bosch & VII-XII & $\mathrm{SC}$ \\
\hline H. dentatum Cav. & VIII-XI & FP \\
\hline H. dicranotrichum (C.Presl) Hook. ex Sadeb & VIII-XI & $\mathrm{V}$ \\
\hline H. ferrugineum Colla & X-XII, JF & FP \\
\hline H. fuciforme $\mathrm{Sw}$. & VII-XII, JF & FP \\
\hline H. krauseanum Phil. & VIII-XI & $\mathrm{SC}$ \\
\hline H. pectinatum Cav. & VII-XII, JF & FP \\
\hline H. peltatum (Poiret) Desv. & IV, VII-XII & $\mathrm{SC}$ \\
\hline H. plicatum Kaulf. & VI-XII, JF & FP \\
\hline H. secundum Hook. et Grev. & $\mathrm{X}-\mathrm{XII}, \mathrm{JF}$ & V \\
\hline H. seselifolium C.Presl & VII-XII & $\mathrm{SC}$ \\
\hline H. tortuosum Hook. et Grev & IX-XII, JF & V \\
\hline H. tumbridgense (L.) Sm. & VII-XI & $\mathrm{SC}$ \\
\hline H. umbratile Diem et J.S.Licht. & $\mathrm{X}$ & $\mathrm{SC}$ \\
\hline Serpyllopsis caespitosa (Gaudich.) C.Chr. & $\mathrm{X}$-XII & $\mathrm{IC}$ \\
\hline
\end{tabular}

De las 18 de especies de Hymenophyllum descritas para Chile continental (Rodríguez 1995), se encontraron 16. Las 2 ausentes son dudosas para Chile, puesto que Hymenophyllum falklandicum Baker, distribuido teóricamente desde la Región del Biobío al sur, es reconocido por Diem \& Lichtenstein (1959) como una especie altamente variable y fácilmente confundible con Hymenophyllum peltatum. Las diferencias entre estas especies están dadas porque la primera presenta una lámina más angosta, pinnas inferiores con menos lacinias y dientes del margen más agudos y con menos soros. Estos autores hacen notar que existen dificultades para reconocer los caracteres diagnósticos, lo que puede provocar que los ejemplares estudiados queden la mayoría como material indeterminado, y sólo un pequeño porcentaje determinado con seguridad.

Por otro lado, Hymenophyllum nahuelhuapiense Diem et J.S.Licht., fue descrito como especie nueva para la región de Nahuel Huapi, Argentina, por Diem \& Lichtenstein 
(1959). No obstante, desde su publicación, estos autores han mencionado que se asemeja tanto en hábito como en su hábitat a Hymenophyllum tumbridgense (L.) Sm., siendo difíciles diferenciarlas, ya que su separación es por la longitud del pecíolo y la lámina, además de la presencia de pelos y las pinas más remotas como caracteres vegetativos. También mencionan que $H$. nahuelhuapiense se confunde con $H$. falklandicum, sugiriendo que éstas puedan no ser realmente especies distintas. Finalmente, los materiales de estas 2 especies conflictivas estudiados en Chile corresponden a un par de ejemplares colectados en Peulla, en la frontera con Nahuel Huapi por su autor J. Diem. Por todos estos antecedentes, se pone en duda la presencia de estos individuos en territorio chileno y hace necesario revisar las bases de datos y colecciones para nuestro país.

Asumiendo lo anterior, cabe destacar que en un área tan pequeña como el Parque Katalapi y su entorno, se encuentra prácticamente la totalidad de las Hymenophyllaceae de Chile continental. Esto se debe, principalmente, a las condiciones del clima que permiten la presencia de un bosque que ha permanecido en el tiempo, favoreciendo el desarrollo de estos helechos. También es notable la importancia que tiene el lugar desde el punto de vista de la conservación, ya que seis especies de Hymenophyllaceae clasificadas como Vulnerables (Baeza et al. 1998), se encuentran en esta área de la Cordillera de Quillaipe.

Además, dada la dificultad de identificación del género Hymenophyllum, surge la necesidad de generar una clave taxonómica que no se base en caracteres reproductivos como las que actualmente existen (características de los soros e indusios) (Diem \& Lichtenstein 1959, Rodríguez 1995). Este tipo de claves taxonómicas deja fuera de posibilidad de identificación a todos aquellos individuos que no se encuentren en un estadio reproductivo.

Sobre la base de los individuos estudiados y a las descripciones originales, se presenta una clave para los representantes presentes en el parque Katalapi de dicho género basada en estructuras vegetativas y constantes entre las especies.

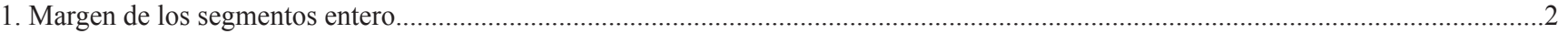

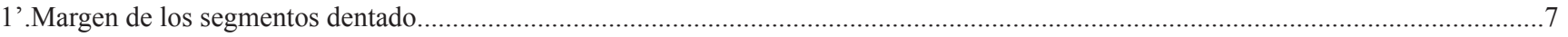

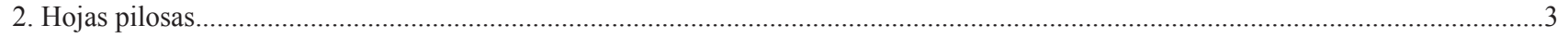

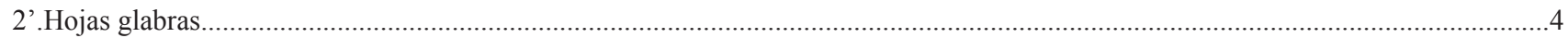

3. Lámina con abundantes pelos cobrizos en ambas superficies.......................................................................... ferrugineum

3'.Lámina generalmente con pelos bifurcados en el margen y superficie inferior...................................................... dicranotrichum

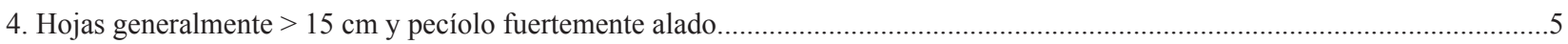

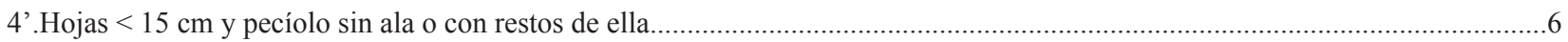

5. Hojas aglomeradas, ala del pecíolo de $1 \mathrm{a} 1,5 \mathrm{~mm}$................................................................................................... fuciforme

5'.Hojas distanciadas, ala del pecíolo de 1,5 a $2 \mathrm{~mm}$........................................................................ caudiculatum var. productum

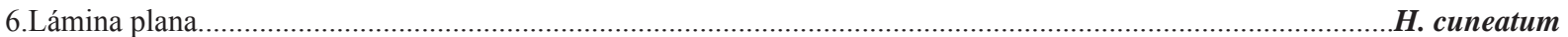

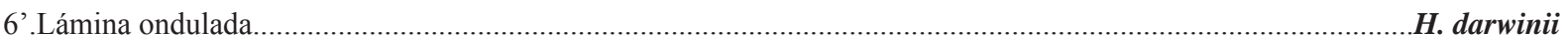

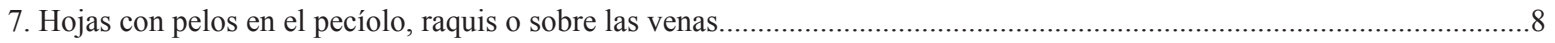

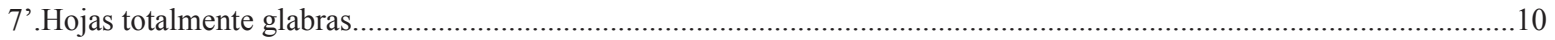

8. Segmentos dirigidos hacia el ápice y hacia la base de la lámina.............................................................. dentatum

8'. Todos los segmentos dirigidos hacia el ápice de la lámina o sólo con los de la pinna basal orientados hacia ambos lados.

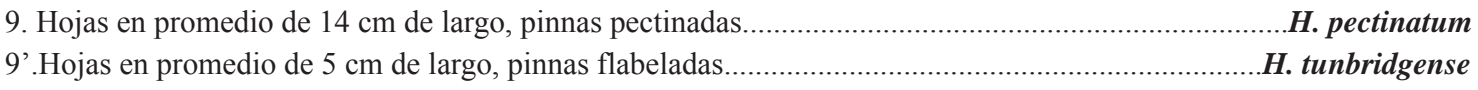

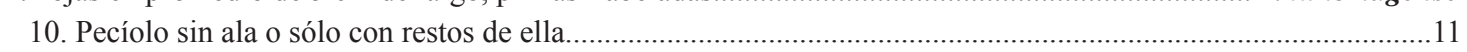

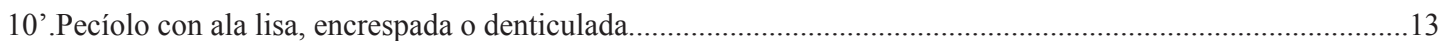

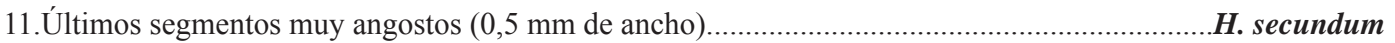

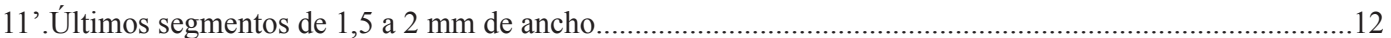

12. Margen de los segmentos suavemente dentado.......................................................................... umbratile

12'.Margen de los segmentos fuertemente dentado............................................................................ peltatum

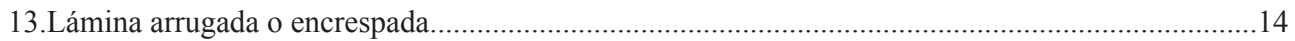

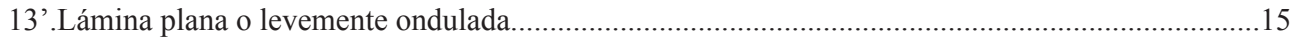

14. Alas del raquis y margen de los últimos segmentos dentado-ciliado........................ tortuosum

14'.Alas del raquis y margen de los últimos segmentos dentado-sinuoso......................... plicatum

15. Pecíolo con ala angosta y lisa.....

H. krauseanum

15 '.Pecíolo con ala gruesa y fuertemente encrespada.

..H. seselifolium 


\section{AGRADECIMIENTOS}

Proyecto FONDECYT 1090397.

\section{BIBLIOGRAFÍA}

Baeza, M., E. Barrera, J. Flores, C. Ramírez \& R. Rodríguez. 1998. Categorías de conservación de Pteridophyta nativas de Chile. Boletín del Museo Nacional de Historia Natural 47: 23-46.

Diem, J. \& J. Lichtenstein. 1959. Las Himenofiláceas del área argentino-chilena del sud. Darwiniana 11: 611-760.

Godoy, R. \& H. FigueroA. 1989. Composition and distribution of the pteridophyte flora of continental and insular Chile. Nova Hedwigia 48: 437-453.

Grau, J. 1995. Aspectos geográficos de la Flora de Chile. En:
C, Marticorena \& R. Rodríguez (eds.) Flora de Chile, Volumen 1, pp. 119-309. Editora Aníbal Pinto S.A. Concepción, Chile.

Ponce, M., K. Mehltreter \& E. Del Sota. 2002. Análisis biogeográfico de la diversidad pteridofítica en Argentina y Chile continental. Revista Chilena de Historia Natural. 75: 703-712.

Reyes-Díaz. M., M. Alberdi, F. Piper, L.A. Bravo \& L.J.Corcuera. 2005. Low temperature responses of Nothofagus dombeyi and Nothofagus nitida, two evergreen species from South Central Chile. Tree Physiology 25:1389-1398.

Rodríguez, R. 1995. Pteridophyta. En: C. Marticorena \& R. Rodríguez (eds.), Flora de Chile, Volumen 1, pp. 119-309. Editora Aníbal Pinto S.A. Concepción, Chile.

Tryon, R.M. \& A.F. Tryon. 1982. Fern and allied plants, with special reference to tropical America. Springer-Verlag, New York. 857 pp.

Recibido: 04.10.11

Aceptado: 29.06.12 